\title{
Poster Abstract: A Neural Networks based Caching Scheme for Mobile Edge Networks
}

\author{
Zhou Qin \\ Rutgers University \\ zq58@cs.rutgers.edu
}

\author{
Yikun Xian \\ Rutgers University \\ yx150@cs.rutgers.edu
}

\author{
Desheng Zhang \\ Rutgers University \\ desheng.zhang@cs.rutgers.edu
}

\begin{abstract}
Mobile edge networks are pervasive now due to the ubiquitous $4 \mathrm{G}$ networks and coming $5 \mathrm{G}$ networks, broad edge computing applications are enabled in the meantime, such as mobile bus WiFi. In this paper, we focus on the caching problem in the mobile edge networks and use bus WiFi as an example to further investigate. Mobile bus WiFi is a newly emerged service in modern cities, which provides convenience for citizens and gains certain benefits for operators via commercial advertisements and other services. While the vital challenge for the bus WiFi industry is the high cost of cellular traffic considering the massive number of users and longtime running hours of the bus system. To tackle this, we investigate the caching problem in a nation-scale bus WiFi network deployed in 22 cities of China with 34,377 WiFi devices. We then delve a fundamental question, i.e., how can historical visiting records help us predict future visiting events and further save the cellular traffic by caching. In detail, we propose a Deep Neural Networks (DNN) based method by considering bus WiFi users' historical visits to cached contents to save cellular traffic data for WiFi providers. We implement our method via the city-scale bus WiFi data and compare with a series of state-of-the-art models, the results show that our method achieves the best performance.
\end{abstract}

\section{CCS CONCEPTS}

- Information systems $\rightarrow$ Location based services; • Networks $\rightarrow$ Cyber-physical networks.

\section{KEYWORDS}

Bus WiFi; user behavior; deep neural networks; cache

\section{ACM Reference Format:}

Zhou Qin, Yikun Xian, and Desheng Zhang. 2019. Poster Abstract: A Neural Networks based Caching Scheme for Mobile Edge Networks. In SenSys '19: Conference on Embedded Networked Sensor Systems, November 10-13, 2019, New York, NY, USA. ACM, New York, NY, USA, 2 pages. https://doi.org/10. $1145 / 3356250.3361961$

\section{INTRODUCTION}

In recent years, we are witnessing a rapid growth of mobile edge networks and related edge computing applications. Among them,

Permission to make digital or hard copies of part or all of this work for personal or classroom use is granted without fee provided that copies are not made or distributed for profit or commercial advantage and that copies bear this notice and the full citation on the first page. Copyrights for third-party components of this work must be honored. For all other uses, contact the owner/author(s).

SenSys '19, November 10-13, 2019, New York, NY, USA

(c) 2019 Copyright held by the owner/author(s).

ACM ISBN 978-1-4503-6950-3/19/11 ..\$15.00

https://doi.org/10.1145/3356250.3361961
WiFi deployment and usage on public transport systems (e.g., subways and buses) are more pervasive due to the convenience for passengers and potential commercial value for operators. For example, there were more than 100,000 WiFi devices deployed on urban transit buses in China by 2015 and it is expected that almost every bus and train will offer WiFi aboard within a few years. Besides, it is reported that there are more than 3.39 million unique users using WiFi on public transport in 9 European cities [3].

As a promising technology, mobile WiFi is beneficial for both passengers and service providers. For passengers, mobile WiFi enables them access to the Internet more conveniently with a lower cost and high-quality service compared to the users' cellular data plan. Hence, mobile WiFi service has the potential to increase ridership by improving user experience on the buses. On the other hand, for the service providers, personalized advertisements can be cast based on user profiling by data collected through WiFi systems, which may potentially increase their revenues. Currently, there are many real-world bus WiFi systems, e.g., Google bus WiFi [1], New York bus WiFi [2], and Shenzhen Huashi WiFi, and most of them are free for bus riders to use.

However, despite the potential benefits of mobile WiFi for both passengers and service providers, mobile WiFi devices currently have not been installed nationwide due to several reasons, e.g., high infrastructure cost, unclear operational and usage patterns, and most importantly, the high operational cost especially the cost of cellular data. Hence, understanding operational patterns and reducing the cost of large-scale mobile WiFi networks are essential to pave ways for further promotion. Currently, the mobile WiFi devices contain hard disks to cache multimedia contents for visiting, in order to save some cellular data. However, a fixed content caching scheme does not work well in the mobile WiFi case because of the unique characteristics of bus WiFi networks, e.g., the high dynamics of user connection and unbalanced usage among different bus lines.

In this paper, we design a sophisticated DNN based scheme to cache contents on bus WiFi devices to improve the system performance of the mobile WiFi network, e.g., reducing traffic data and connection delay. In details, we approach the problem from the individual user perspective by considering historical visited contents of passengers, then we treat the problem as a prediction problem and construct the DNN based method for it. The method is then evaluated by the bus WiFi log data in the Chinese city Shenzhen.

\section{SYSTEM DESIGN}

\subsection{Data Description}

The data are collected from the WiFi system by three types according to different generation sources, i.e., static data, platform data, and device data. The static data contain information about the basic properties of the device and other affiliated information, which 
were generated when the device was produced and installed. This type of data is relatively small and static. The platform data are generated on the platform side when WiFi devices communicate with platforms, which can only be obtained from the platforms. The largest data are device data, which are collected by WiFi devices and stored locally on the devices.

In particular, the static data include information when devices are produced and installed, such as the ID of the device, operators, affiliated regions, supportive web types (e.g., 2G, 3G, and 4G), installation time of the SIM card, province, city, bus line, bus plate number, bus company, and the time of the deployment. Platform data originate from the device level and user level: device-level data include the device ID, starting online time and ending offline time; user-level data include basic information such as user ID, account number, user device type, device ID, starting time and ending time of registration. Device data are generated and temporarily stored on the device end, which include the location of the device, device probes, the data flow of the device; user-related data include connection and disconnection time, the data traffic uploaded and downloaded, portal visiting records, HTTP visiting records, and non-HTTP visiting records. A visualization of WiFi usage heat map is shown in Figure 1.

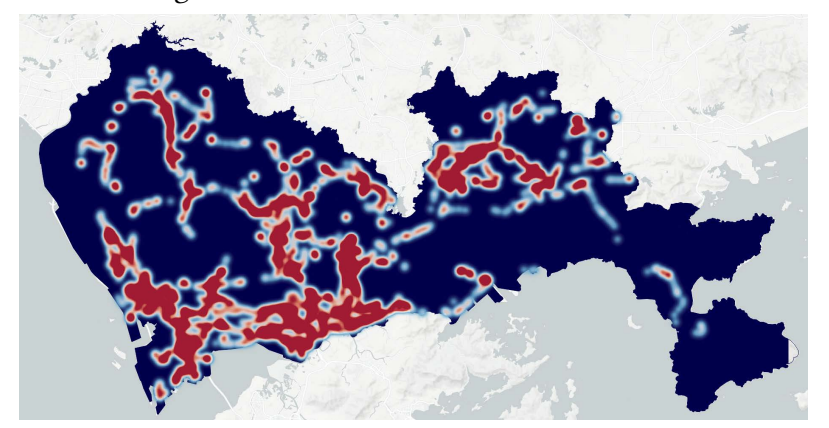

Figure 1: Bus WiFi Usage Distribution

\subsection{System Framework}

The framework of our proposed method is shown in Figure 2. In the bus WiFi scenario, the users visit different contents either stored in the WiFi devices on the bus or Internet via cellular networks. Considering this, we take users' IDs and contents (encoded as itemID) as inputs for our framework. The IDs are hashed into unique integers. Considering the massive number of users and contents involved int the bus WiFi application, we first implement neural network embedding upon the userID and itemID by the size of 50 to better represent the data in the transformed space. With the $n \times 50$ ( $n$ is the number of IDs) size data embedded, we then construct fully connected layers (FC in Figure 2) to encode the embedded data. After this, we concatenate the data from the userID and itemID. Two layers of fully connected layers are applied upon the above outputs. We also consider Dropout in the layers to reduce the possibility of overfitting the training data. In the end, we compare the predicted visiting contents (itemID) to the true itemID to show the performance.

\section{EVALUATION RESULTS}

We evaluate our method by the bus WiFi data from the Chinese city Shenzhen. Here we directly predict the contents (itemID) based

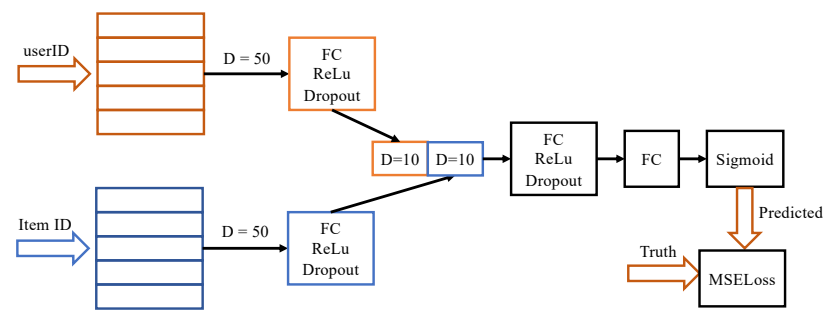

Figure 2: System Framework

on historical data, we select all users who have ten days of usage data from the two-month data. Then we use the previous 9 days data to predict the visiting of last day. All contents including local contents and Internet URLs are considered. For each user during a specific day, we calculate the corresponding visiting frequencies. Then our goal is to predict what are the corresponding visiting contents and how many times does a user visit them for the given date. For the results, we consider two concrete metrics, i.e., Root Mean Square Error (RMSE) and Mean Absolute Error (MAE) to quantify the performance. Once we correctly predict the URLs the users may visit and the corresponding visiting frequency, we then can cache contents based on the prediction results, thus traffic data can be saved when users visit the cached contents.

We implement our proposed method with Pytorch and run on NVIDIA K80. The learning rate is set to be 0.001 and the batch size is set as 64. The model runs for 30 epochs. We adopt Adam optimizer here and the results are shown in Table 1. As we can see, our proposed model achieves satisfying results.

Table 1: Evaluation Results

\begin{tabular}{lll}
\hline Methods & RMSE & MAE \\
\hline NormalPredictor & 0.2222 & 0.1518 \\
KNN & 0.1575 & 0.0766 \\
KNNWithMeans & 0.1569 & 0.0856 \\
SVD & 0.1598 & 0.0957 \\
NMF & 0.1637 & 0.0947 \\
CoClustering & 0.1996 & 0.118 \\
Our work & 0.1516 & 0.0771 \\
\hline
\end{tabular}

\section{CONCLUSION}

In this paper, we propose a dedicated DNN based scheme for caching contents on mobile WiFi devices to save traffic data for WiFi network operators by considering users behaviors, which has the potential to help them to reduce operational cost, encourage future system upgrading, and deploy bus WiFi networks in more cities. We evaluate our method via the bus WiFi log data in one city, the results show our method achieves the best performance compared to state-of-the-art methods. The accurate prediction can then benefit the service providers since effective caching can be implemented and the users since cached contents can provide more stable and higher speed accessing.

\section{REFERENCES}

[1] Mikhail Afanasyev, Tsuwei Chen, Geoffrey M Voelker, and Alex C Snoeren. 2010. Usage patterns in an urban WiFi network. IEEE/ACM Transactions on Networking (TON) 18, 5 (2010), 1359-1372.

[2] David P Blinn, Tristan Henderson, and David Kotz. 2005. Analysis of a Wi-Fi hotspot network. In Papers presented at the 2005 workshop on Wireless traffic measurements and modeling. USENIX Association, 1-6.

[3] Monica Ferrari. 2017. Benefits of free on-board Wi-Fi for public transport. https: //www.tanaza.com/blog/benefits-of-free-on-board-wi-fi-for-public-transport/. 